\title{
AVALIAÇÃO DOS EFEITOS DO CONSUMO DO COGUMELO AGARICUS BLAZEI EM INDICADORES DE QUALIDADE DE VIDA DE IDOSOS
}

\author{
Luiz Julio Rocha de Oliveira* \\ Juliana Ladeira Garbaccio* \\ Wiliam César Bento Regis ${ }^{* *+}$
}

\begin{abstract}
RESUMO: Objetivou-se avaliar a relação entre 0 uso do cogumelo Agaricus blazei, a prática de atividade física e a qualidade de vida entre idosos. Tratou-se de um estudo transversal, realizado com 44 idosos, divididos em grupos: grupo A praticantes de atividade física exclusivamente 21 indivíduos; grupo B - usuários do cogumelo Agaricus blazei e não praticantes de atividade física - 12 indivíduos; grupo C usuários do cogumelo Agaricus blazei e praticantes de atividade física 11 indivíduos. Utilizou-se um questionário estruturado, sendo a análise descritiva realizada no software estatístico SPSS, versão 17.0 grupo $\mathrm{C}$ apresentou maior número de indivíduos que afirmaram possuir sensação de saúde boa/ muito boa, percepção da própria memória muito boa/ boa e, com a menor ocorrência de sintomas de dores, rigidez e edema nas articulações. Assim, sugere-se que 0 uso sinérgico do cogumelo Agaricus blazei com a prática de atividade física pode melhorar a qualidade de vida de idosos.
\end{abstract}

PALAVRAS-CHAVE: Nutrição; Qualidade de vida; Atividade física; Idoso; Agaricus blazei.

\section{EFFECTS ON CONSUMPTION OF MUSHROOM AGARICUS BLAZEI ON ELDERLY'S LIFE QUALITY INDEXES}

\begin{abstract}
The relationship between the use of the mushroom Agaricus blazei, physical activities and life quality among the elderly is investigated. Current transversal study was undertaken with 44 elderly people divided into two groups: Group A - 21 elderly people who practiced physical activity exclusively; Group B - 12 elderly people who used Agaricus blazei but did not practice any physical activity; Group C - 11 elderly people who consumed Agaricus blazeiand practiced physical activity. A structured questionnaire was employed and descriptive analysis was performed with SPSS 17. Group C had the greatest number of elderly people who declared good and very good health, good and very good memory and lower occurrence of pain, rigidity and edema in their joints. The synergic use of the mushroom Agaricus blazei with physical activities may improve the life quality of elderly people.
\end{abstract}

KEYWORDS: Nutrition; Life quality; Physical activity; Elderly people; Agaricus blazei.

\section{INTRODUÇÃo}

0 cogumelo Agaricus blazei possui comprovadas propriedades medicinais, como o estímulo do sistema imunológico, efeitos anticancerígenos, como agente antihipercolesterolêmicos e hepatoprotetor, atividade antiviral (anti HIV) e atua na melhoria do efeito tóxico da quimioterapia e radioterapia (RÉGIS et al., 2015; HETLAND et al., 2008; WANG et al., 2014; MIRANDA et al., 2014; FACCIN et al., 2007).

Estudos apontam que a prática de atividade física induz a atividade das enzimas antioxidantes, potencializando a atividade mitocondrial, principalmente na população com sobrepeso/obesa (FARINHA et al., 2014; ROWISKI et al., 2013). Adicionalmente, 0 exercício físico promove a alteração do quadro de re-

\footnotetext{
Acadêmico de Nutrição da Pontifícia Universidade Católica de Minas Gerais (PUC Minas), Brasil.

** Docente associada à Pontifícia Universidade Católica de Minas Gerais (PUC Minas), Brasil.

*** Docente no Programa de Pós-Graduação em Biologia de Vertebrados na Pontifícia Universidade Católica de Minas Gerais (PUC Minas), Programa de Pós-Graduação em Nutrição e Saúde da Universidade Federal de Minas Gerais (UFMG) e no Programa de Pós-Graduação em Medicina Tropical e Infectologia da Universidade Federal de Minas Gerais (UFMG), Brasil.
} 
sistência à insulina, bem como no perfil lipídico, tendo também potencial impacto na redução da glicemia (MARINHO et al., 2015).

Sabe-se que a prática de atividades físicas diminui o estresse oxidativo aumentado em decorrência do envelhecimento, também apresentando efeitos benéficos no tratamento da depressão e na prevenção de doenças crônicas não transmissíveis, principalmente no que tange ao combate à obesidade, ao diabetes e às doenças cardíacas (ROWISKI et al., 2013; PENTECOST et al., 2015; PAOLI; BIANC0; 2015).

Neste sentido, elencou-se como pergunta norteadora desta pesquisa: existe alguma relação entre a prática de atividades físicas e ingestão do cogumelo Agaricus blazei na qualidade de vida de idosos?

0 envelhecimento é um fato iminente e agudo no Brasil, onde há uma alteração clara na pirâmide etária, com aumento significativo da população idosa (IBGE, 2011). Almeja-se, no âmbito das discussões que envolvem o idoso, promover envelhecimento com qualidade de vida e esta temática envolve inúmeros segmentos da sociedade, inclusive na pesquisa de novos produtos e na relação com atividades físicas.

Neste estudo objetivou-se avaliar a relação entre 0 uso do cogumelo Agaricus blazei, a prática de atividade física e a qualidade de vida entre idosos.

Importante ressaltar que o cogumelo Agaricus blazei, utilizado como suplemento pelos entrevistados no presente estudo, possui a seguinte composição de macronutrientes por 100 gramas:

Quadro 1. Centesimal de composição do gogumelo Agaricus blazei

\begin{tabular}{|l|c|c|}
\hline $\begin{array}{l}\text { Macronutri- } \\
\text { entes }\end{array}$ & $\begin{array}{c}\text { Quantidade por } \mathbf{1 0 0} \\
\mathbf{g}\end{array}$ & Método \\
\hline Fibras & $4,85 \mathrm{~g}$ & $\begin{array}{c}\text { Enzymatic } \\
\text { Gravimetric }\end{array}$ \\
\hline Gorduras totais & $3,66 \mathrm{~g} ;$ & IAL 032-34/IV \\
\hline Proteínas & $38,33 \mathrm{~g}$ & IAL 451/IV \\
\hline Umidade & $7,12 \mathrm{~g}$ & IAL 012/IV \\
\hline Beta-glucana & $14.28 \%$ & $\begin{array}{c}\text { A0AC 0fficial } \\
\text { 992.28 }\end{array}$ \\
\hline
\end{tabular}

Fonte: Rotulagem do produto EnergyVita, empresa Minasfungi.

\section{MATERIAIS E MÉTODOS}

Tratou-se de uma pesquisa transversal realizada em Belo Horizonte/MG, com idosos de ambos os sexos, lúcidos e capazes de responder às perguntas $\mathrm{e}$ residentes apenas em domicílios, sendo excluídos os internos de qualquer tipo de instituição hospitalar ou de longa permanência.

Utilizou-se de contato telefônico, por um único entrevistador, para que os idosos respondessem a um questionário estruturado contendo 81 questões referentes a aspectos baseados no Inquérito sobre Saúde, Bem-Estar e Envelhecimento - Sabe (UNIVERSIDADE DE SÃO PAUL0, 2006), coordenado pela Organização Pan-Americana de Saúde (OPAS/OMS), e saúde (doenças, prática de atividades físicas, consumo de produtos naturais).

A anuência para participar da pesquisa foi fornecida pelos participantes por meio da assinatura do Termo de Consentimento Livre e Esclarecido TCLE, entregue pessoalmente, ou com consentimento verbal após a leitura do TCLE, via contato telefônico. A pesquisa foi aprovada por Comitê de Ética em Pesquisa CAAE: 15431913.9.0000.5137 sob o parecer $n$. 289.951.

As respostas foram categorizadas e analisadas descritivamente no programa statistical package for the social sciences (Spss/pc) versão 13.0. Utilizou-se 0 teste qui-quadrado e 0 teste de Fisher para validar a significância $(p<0,05)$ da associação entre as variáveis sociodemográficas, de saúde dos idosos, prática de atividade física e consumo do cogumelo.

$\mathrm{Na}$ análise, os idosos foram categorizados em três grupos:

- Grupo A - idosos praticantes de atividade física exclusivamente - 21 indivíduos;

- Grupo B - idosos usuários do cogumelo Agaricus blazei e não praticantes de atividade física - 17 indivíduos;

- Grupo C - idosos usuários do cogumelo Agaricus blazei e praticantes de atividade física - 17 indivíduos. 
A ingestão do cogumelo Agaricus blazei, grupos $\mathrm{B}$ e $\mathrm{C}$, se deu mediante o consumo do cogumelo in natura, triurado, em cápsulas, via suplemento EnergyVita, com a dosagem média de quatro cápsulas por dia, sendo que cada cápsula possui 400 mg de cogumelo Agaricus blazei triturado.

Cabe ressaltar que os usuários do cogumelo Agaricus blazei o consumiam há pelo menos um ano.
No que tange à prática de atividades físicas, grupo A, considerou-se qualquer tipo de atividade mecânica que vise a melhora da perfomance física dos praticantes, desde que realizada com a frequência mínima de duas vezes por semana.

Tabela 1. Dados sociodemográficos dos questionários estruturados colhidos dos idosos

\begin{tabular}{|c|c|c|c|c|}
\hline \multicolumn{5}{|c|}{ DADOS SOCIODEMOGRÁFICOS DOS QUESTIONÁRIOS ESTRUTURADOS COLHIDOS DOS IDOSOS } \\
\hline Variáveis & $\begin{array}{c}\text { Grupo } A(N=21) \\
n(\%)\end{array}$ & $\begin{array}{c}\text { Grupo B (N=12) } \\
n(\%)\end{array}$ & $\begin{array}{c}\text { Grupo C (N=11) } \\
n(\%)\end{array}$ & Total - N (\%) \\
\hline \multicolumn{5}{|l|}{ Sexo } \\
\hline Feminino & $17(81,0)$ & $9(75,0)$ & $11(100)$ & $37(84,1)$ \\
\hline Masculino & $4(19,0)$ & $3(25,0)$ & - & $7(15,9)$ \\
\hline \multicolumn{5}{|l|}{ Idade } \\
\hline 60-69 anos & $21(100)$ & $7(58,3)$ & $6(54,5)$ & $34(77,3)$ \\
\hline 70-79 anos & - & $3(25)$ & $4(36,4)$ & $7(15,9)$ \\
\hline 80-89 anos & - & $2(16,7)$ & $1(9,1)$ & $3(6,8)$ \\
\hline \multicolumn{5}{|l|}{ Cor da Pele } \\
\hline Parda & $11(52,4)$ & $5(41,7)$ & $5(45,5)$ & $21(47,7)$ \\
\hline Branca & $4(19,0)$ & $7(58,3)$ & $5(45,5)$ & $16(36,4)$ \\
\hline Preta & $6(28,6)$ & - & - & $6(13,6)$ \\
\hline Amarela & - & - & $1(9,1)$ & $1(2,3)$ \\
\hline \multicolumn{5}{|l|}{ Local de Nascimento } \\
\hline Zona Rural & $13(61,9)$ & $5(41,7)$ & $6(54,5)$ & $24(54,5)$ \\
\hline Zona Urbana & $8(38,1)$ & $7(58,3)$ & $5(45,5)$ & $20(45,5)$ \\
\hline \multicolumn{5}{|c|}{ Exerce algum trabalho remunerado } \\
\hline Não & $17(81,0)$ & $9(75,0)$ & $9(81,8)$ & $35(79,5)$ \\
\hline Sim & $4(19,0)$ & $3(25,0)$ & $2(18,2)$ & $9(20,5)$ \\
\hline
\end{tabular}

Fonte: Dados da pesquisa.

\section{RESULTADOS}

Entrevistaram-se 55 idosos, 37 (84,1\%) do sexo feminino, $21(47,7 \%)$ declararam-se com a pele de cor parda, $24(54,5 \%)$ afirmaram ter nascido em zona rural, 35 (79,5\%) não exercer qualquer tipo de trabalho.
Quando indagados sobre a própria saúde observou-se que a sensação de saúde boa ou muito boa foi significativamente maior no grupo $\mathrm{C}(72,7 \%)$, seguido do grupo A $(57,1 \%)$ e do grupo B $(50 \%)$ (Tabela 2).

No que tange à percepção da própria memória, $72,7 \%$ dos participantes do grupo $\mathrm{C}$ afirmaram 
apresentar memória muito boa ou boa, seguido do grupo A (50\%) e B $(47,6 \%)$. Apesar dos entrevistados do grupo $B$ não relatarem melhora no quadro de hipertensão arterial, verificou-se que a sinergia entre 0 uso do cogumelo Agaricus blazei e a prática de atividade física possivelmente contribuiu positivamente no controle da hipertensão arterial (Tabela 2).

Quanto aos sintomas de dores, rigidez e edema nas articulações, os participantes do grupo C (usuários do cogumelo Agaricus blazei e praticantes de atividade física) referiram menor ocorrência deles. Não houve expressiva variação na proporção de usuários de produtos naturais fitoterápicos e vitamina $\mathrm{C}$, entretanto, para o consumo de óleo de peixe, verificou-se adesão somente pelos integrantes do grupo C (Tabela 2).

No que tange aos entrevistados usuários do cogumelo Agaricus blazei (grupos B e C), verificou-se que esses relataram maior frequência urinária $\mathrm{e}$ de evacuação após o seu uso, demonstrando possível efeito diurético e de melhora na motilidade intestinal, bem como relataram maior sentimento de calmaria, tranquilidade, maior regularidade no sono e maior disposição (Tabela 5).

Esses resultados sugerem que 0 uso do cogumelo Agaricus blazei (grupos B e C) possibilitou maior frequência urinária e motilidade intestinal nos

Tabela 2. Respostas ao questionário estruturado aos questionários estruturados colhidos dos idosos, especificamente quanto aos quesitos: percepção da própria saúde; memória; hipertensão arterial sistêmica (HAS); análise das HAS em 12 meses; dores, rigidez, edema nas articulações; uso de óleo de peixe; uso de fitoterápico; uso de vitamina C

\begin{tabular}{|c|c|c|c|}
\hline \multicolumn{4}{|c|}{ DADOS DOS QUESTIONÁRIOS ESTRUTURADOS COLHIDOS DOS IDOSOS } \\
\hline Variáveis & $\begin{array}{c}\text { Grupo } A(N=21) \\
n(\%)\end{array}$ & $\begin{array}{c}\text { Grupo B (N=12) } \\
n(\%)\end{array}$ & $\begin{array}{c}\text { Grupo C (N=11) } \\
\text { n (\%) }\end{array}$ \\
\hline \multicolumn{4}{|l|}{ Percepção da própria saúde } \\
\hline Muito boa/ boa & $12(57,1)$ & $6(50)$ & $8(72,7)$ \\
\hline Regular & $9(43,9)$ & $6(50)$ & $2(18,2)$ \\
\hline Ruim/ muito ruim & - & - & $1(9,1)$ \\
\hline \multicolumn{4}{|l|}{ Memória } \\
\hline Muito boa/ boa & $10(47,6)$ & $6(50)$ & $8(72,7)$ \\
\hline Regular & $10(47,6)$ & $6(50)$ & $2(18,2)$ \\
\hline Ruim/ muito ruim & $1(4,8)$ & - & $1(9,1)$ \\
\hline \multicolumn{4}{|c|}{ Hipertensão arterial sistêmica (HAS) } \\
\hline Possui & $19(90,4)$ & $8(66,7)$ & $8(72,7)$ \\
\hline Não possui & $2(9,6)$ & $4(33,3)$ & $3(27,3)$ \\
\hline \multicolumn{4}{|l|}{ Análise da HAS em 12 meses } \\
\hline Melhor & $11(57,9)$ & - & $5(62,5)$ \\
\hline Sem alteração & $7(36,8)$ & $8(100)$ & $3(37,5)$ \\
\hline Pior & $1(5,3)$ & - & - \\
\hline \multicolumn{4}{|c|}{ Dores, rigidez, edema nas articulações } \\
\hline Possui & $14(66,7)$ & $8(66,7)$ & $5(45,5)$ \\
\hline Não possui & $7(33,3)$ & $4(33,3)$ & $6(54,5)$ \\
\hline \multicolumn{4}{|l|}{ Uso de óleo de peixe } \\
\hline $\operatorname{Sim}$ & - & - & $6(54,5)$ \\
\hline Não & $21(100)$ & $12(100)$ & $5(45,5)$ \\
\hline \multicolumn{4}{|l|}{ Uso de fitoterápico } \\
\hline $\operatorname{Sim}$ & $9(42,9)$ & $6(50,0)$ & $6(54,5)$ \\
\hline Não & $12(57,1)$ & $6(50,0)$ & $5(45,5)$ \\
\hline \multicolumn{4}{|l|}{ Uso de vitamina C } \\
\hline $\operatorname{Sim}$ & $3(14,3)$ & $1(8,3)$ & $1(9,1)$ \\
\hline Não & $18(85,7)$ & $11(91,7)$ & $10(90,9)$ \\
\hline
\end{tabular}

Fonte: Dados da pesquisa. 
usuários, gerando melhora no desempenho de suas funções orgânicas regulares, tendo, como consequência, melhora na regularidade do respectivo sono, o que gerou maior disposição para 0 desenvolvimento das tarefas do dia a dia desses idosos, bem como mais calma em suas vidas.

Tabela 3. Todos os idosos que fazem uso de cogumelo Agaricus blazei

\section{DADOS SOCIODEMOGRÁFICOS DOS IDOSOS DOS QUES- TIONÁRIOS ESTRUTURADOS COLHIDOS RELACIONADOS AO USO DO COGUMELO}

\begin{tabular}{lcccc}
\hline & \multicolumn{4}{c}{ Faz uso do cogumelo? } \\
\hline & Sim & Não & Total & $P$ \\
\hline Idade & & & & \\
\hline $60-69$ anos & 13 & 21 & 34 & 0,03 \\
$70-79$ anos & 7 & - & 7 & \\
80-89 anos & 3 & - & 3 & \\
\hline Cor da pele & & & & \\
\hline Branca & 12 & 4 & 16 & 0,007 \\
Parda-amarela & 11 & 11 & 22 & \\
Preta & - & 6 & 6 & \\
\hline Total & 23 & 21 & 44 & \\
\hline
\end{tabular}

Fonte: Dados da pesquisa.

Tabela 4. Dos participantes do grupo C, cabe ressaltar os seguintes quesitos dos respectivos formulários

(continua)

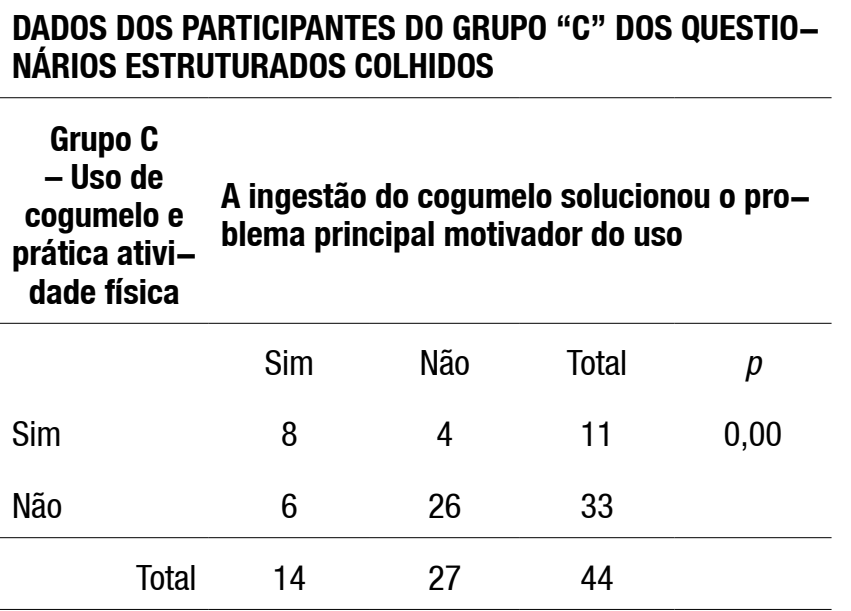

(conclusão)

DADOS DOS PARTICIPANTES DO GRUPO "C" DOS QUESTIONÁRIOS ESTRUTURADOS COLHIDOS

Grupo C

- Uso de cogumelo e prática ativi-

A ingestão do cogumelo solucionou o problema principal motivador do uso dade física

Aumento da disposição para atividades após ingestão do cogumelo

\begin{tabular}{|c|c|c|c|c|}
\hline & Sim & $\begin{array}{c}\text { Sem } \\
\text { alteração }\end{array}$ & Total & $p$ \\
\hline Sim & 8 & 3 & 11 & 0,01 \\
\hline Não & 5 & 28 & 33 & \\
\hline Total & 13 & 31 & 44 & \\
\hline
\end{tabular}

Faz uso concomitante de óleo de peixe

\begin{tabular}{|c|c|c|c|c|}
\hline & Sim & Não & Total & $p$ \\
\hline Sim & 6 & 5 & 11 & 0,00 \\
\hline Não & - & 33 & 33 & \\
\hline Total & 6 & 38 & 43 & \\
\hline
\end{tabular}

Fonte: Dados da pesquisa.

Tabela 5. Respostas dos entrevistados usuários do cogumelo Agaricus blazei (Grupos B e C) quanto à pergunta

(continua)

DADOS DOS PARTICIPANTES DO GRUPO "B" $\mathrm{e}$ "C" ACERCA DA REAÇÃO APÓS A INGESTÃO DO COGUMELO Agaricus blazei

Após a ingestão do cogumelo Agaricus (Cogumelo do Sol, Energyvita...) sentiu-se:

\begin{tabular}{lcc}
\hline Opções de Resposta & $\begin{array}{c}\text { Percentual de } \\
\text { Resposta }\end{array}$ & $\begin{array}{c}\text { Número de } \\
\text { Respostas }\end{array}$ \\
\hline Mais disposto(a) & $66,7 \%$ & 14 \\
Com o sono mais regular & $28,6 \%$ & 6 \\
$\begin{array}{l}\text { Urinando com mais } \\
\text { frequência }\end{array}$ & $19,0 \%$ & 4 \\
$\begin{array}{l}\text { Evacuando com mais } \\
\text { frequência }\end{array}$ & $23,8 \%$ & 5 \\
Mais calmo(a) & $19,0 \%$ & 4 \\
Mais agitado(a) & $14,3 \%$ & 3 \\
\hline
\end{tabular}


(conclusão)

DADOS DOS PARTICIPANTES DO GRUPO "B" $\mathrm{e}$ "C" ACERCA DA REAÇÃO APÓS A INGESTÃO DO COGUMELO Agaricus blazei

Após a ingestão do cogumelo Agaricus (Cogumelo do Sol, Energyvita...) sentiu-se:

\begin{tabular}{lcc}
\hline Opções de Resposta & $\begin{array}{c}\text { Percentual de } \\
\text { Resposta }\end{array}$ & $\begin{array}{c}\text { Número de } \\
\text { Respostas }\end{array}$ \\
\hline Com a libido aumentada & $0,0 \%$ & - \\
Outro (especifique) & $52,4 \%$ & 11 \\
\hline
\end{tabular}

Fonte: Dados da pesquisa.

Tabela 6. Respostas dos entrevistados usuários do cogumelo Agaricus blazei (Grupo C) que praticam atividade física, quanto à pergunta

DADOS DOS PARTICIPANTES DO GRUPO "C" ACERCA DA REAÇÃO APÓS A INGESTÃO DO COGUMELO Agaricus blazei

Após a ingestão do cogumelo Agaricus (Cogumelo do Sol, Energyvita...) sentiu-se:

\begin{tabular}{lcc}
\hline Opções de Resposta & $\begin{array}{c}\text { Percentual de } \\
\text { Resposta }\end{array}$ & $\begin{array}{c}\text { Número de } \\
\text { Respostas }\end{array}$ \\
\hline Mais disposto(a) & $81,8 \%$ & 9 \\
Com o sono mais regular & $45,5 \%$ & 5 \\
$\begin{array}{l}\text { Urinando com mais } \\
\text { frequência }\end{array}$ & $36,4 \%$ & 4 \\
$\begin{array}{l}\text { Evacuando com mais } \\
\text { frequência }\end{array}$ & $45,5 \%$ & 5 \\
Mais calmo(a) & $18,2 \%$ & 2 \\
Mais agitado(a) & $27,3 \%$ & 3 \\
$\begin{array}{l}\text { Com o raciocínio mais } \\
\text { rápido }\end{array}$ & $45,5 \%$ & 5 \\
Com a memória melhor & $18,2 \%$ & 2 \\
Com a libido aumentada & $0,0 \%$ & - \\
Outro (especifique) & $45,5 \%$ & 5 \\
\hline
\end{tabular}

Responderam à pergunta

Fonte: Dados da pesquisa.
Tabela 7. Respostas dos entrevistados usuários do cogumelo Agaricus blazei (Grupos B e C) que NÃO praticam atividade física, quanto à pergunta

DADOS DOS PARTICIPANTES DO GRUPO "C" ACERCA DA REAÇÃO APÓS A INGESTÃO DO COGUMELO Agaricus blazei

Após a ingestão do cogumelo Agaricus (Cogumelo do Sol, Energyvita...) sentiu-se:

\begin{tabular}{lcc}
\hline Opções de Resposta & $\begin{array}{c}\text { Percentual de } \\
\text { Resposta }\end{array}$ & $\begin{array}{c}\text { Número de } \\
\text { Respostas }\end{array}$ \\
\hline Mais disposto(a). & $50,0 \%$ & 5 \\
Com o sono mais regular. & $10,0 \%$ & 1 \\
$\begin{array}{l}\text { Urinando com mais } \\
\text { frequência. }\end{array}$ & $0,0 \%$ & - \\
$\begin{array}{l}\text { Evacuando com mais } \\
\text { frequência. }\end{array}$ & $0,0 \%$ & - \\
Mais calmo(a). & $20,0 \%$ & 2 \\
Mais agitado(a). & $0,0 \%$ & - \\
$\begin{array}{l}\text { Com o raciocínio mais } \\
\text { rápido. }\end{array}$ & $20,0 \%$ & 2 \\
Com a memória melhor. & $10,0 \%$ & 1 \\
Com a libido aumentada. & $0,0 \%$ & - \\
Outro (especifique) & $60,0 \%$ & 6 \\
\hline
\end{tabular}

Responderam à pergunta

Fonte: Dados da pesquisa.

\section{DISCUSSÃO}

Dentre os indivíduos que foram entrevistados no presente estudo percebe-se a prevalência de mulheres.

Pode-se atribuir essa prevalência feminina ao fato de que a maioria dos idosos entrevistados encontra-se na faixa etária de 60 a 69 anos, sendo que, nessa faixa, segundo o censo demográfico de 2010 do Instituto Brasileiro de Geografia e Estatística - IBGE há mais mulheres do que homens, na proporção de 93.252 mulheres para 68.949 homens.

0 grupo, cujos integrantes idosos afirmaram praticar atividade física, apresentou respostas ao 
questionário que indicaram melhora na qualidade de vida. Igualmente na literatura, um estudo com 391 jovens mulheres iranianas demonstrou a existência benéfica da prática de atividade física com a sensação de bem-estar e saúde (SALEHI et al., 2015).

Adicionalmente foi avaliado o uso individual do cogumelo Agaricus blazei e a este também foi reportada melhora na sensação de bem-estar e saúde, memória e alívio de dores. Quando houve a associação entre prática de atividade física e 0 consumo regular do cogumelo Agaricus blazei, os aspectos analisados nesta pesquisa foram bem avaliados ou apresentados pelos participantes. Na literatura não foram encontrados estudos prévios, até o momento, acerca da relação entre a ingestão de cogumelo Agaricus blazei e a sensação de bem-estar.

Com fulcro nos resultados apresentados nos questionários estruturados, verificou-se que 0 uso cumulado do cogumelo Agaricus blazei com a prática de atividade física gerou, para os indivíduos entrevistados, um acréscimo de 15,58 a 22,72 no percentual da sensação de bem-estar e saúde, conforme se verifica nas Tabelas 1 e 2 acima citadas.

Em relação à hipertensão arterial já é bastante estabelecida a benéfica associação da prática de atividade física e 0 controle da hipertensão arterial, (SHIROMA; LEE; 2010) e, em estudos laboratoriais houve melhora na pressão arterial em cobaias que foram submetidos à ingesta do cogumelo Agaricus blazei (HIROKAZU et al., 2014).

Ao se ater aos entrevistados usuários do cogumelo Agaricus blazei (grupos B e C), especificamente quanto às respostas à pergunta: "Após a ingestão do cogumelo Agaricus (Cogumelo do Sol, Energyvita...) sentiu-se:", verificou-se que apenas os citados usuários que concomitantemente praticavam atividade física declararam maior frequência urinária e motilidade intestinal, bem como percentual significativamente maior de regularidade de sono e de maior disposição, conforme se verificam nas Tabelas 6 e 7 .

Baseando-se nos questionários estruturados também é possível aferir que, para os idosos entrevistados, o grupo C, que utilizou o cogumelo Agaricus blazei e praticou atividade física conjunta- mente, apresentou melhora da hipertensão arterial, no período de 12 meses, em percentual 4,61 a 62,50 se comparado ao uso isolado, o que reforça os benefícios do uso cumulado no aumento da qualidade de vida dos idosos.

Outro dado interessante refere-se ao menor relato de dores, rigidez e edema nas articulações vinculado à prática de atividades físicas e uso do cogumelo. Estudo com crianças diagnosticadas com artrite idiopática juvenil revelou uma relação inversamente proporcional entre a prática de atividade física e a dor das articulações (LIMENIS; GROSBEIN; FELDMAN; 2014). Não foram encontrados estudos prévios ao presente acerca da relação entre a ingestão de cogumelo Agaricus blazei e as dores, rigidez e edema nas articulações.

No presente estudo observou-se que 0 uso conjunto do cogumelo Agaricus blazei com a prática de atividade física implicou em melhora nas dores, rigidez e inchaço nas articulações, representando diminuição de 21,22 do percentual de indivíduos com esses sintomas.

Um estudo com 47 idosos concluiu que pouca atividade física pode ser um fator de risco para 0 declínio cognitivo de seres humanos e que a função cognitiva pode ser melhorada pela atividade física (TANIGAWA et al., 2014).

Observando-se os questionários estruturados colhidos no presente estudo, verificou-se que para os idosos aqui entrevistados, a sinergia entre 0 uso do cogumelo Agaricus blazei com a prática de atividade física gerou um aumento de 22,72 a 25,10 no percentual de entrevistados com memória muito boa ou boa, o que sugere a melhora da memória dos idosos com uso concomitante do cogumelo e a prática de atividade física.

0 uso simultâneo do cogumelo Agaricus blazei com a prática de atividade física demonstrou melhora na qualidade de vida dos idosos no sentido de gerar maior cuidado destes com a própria saúde, haja vista que apenas os entrevistados do grupo $\mathrm{C}$ utilizavam 0 óleo de peixe, o que mostra maior disposição destes com a melhora de sua saúde, o que implica em melhora da respectiva qualidade de vida. 
Importante ressaltar que foram realizadas buscas de estudo prévio que relacione a ingestão de cogumelo Agaricus blazei e a memória, no entanto não logou-se êxito.

\section{REFERÊNCIAS}

FACCIN, L.C.; BENATI, F.; RINCÃO, V.P.; MANTOVANI, M.S.; SOARES, S.A.; GONZAGA, M.L.; NOZAWA, C.; CARVALHO LINHARES, R.E. Antiviral activity of aqueous and ethanol extractsand of an isolated polysaccharide from Agaricus brasiliensis against poliovirus type 1. The Society for Applied Microbiology, Letters in Applied Microbiology, 2007. Disponível em: $\quad<$ http://onlinelibrary.wiley.com/doi/10.1111/j. 1472-765X.2007.02153.x/epdf>. Acesso em: 19 ago. 2015.

FARINHA, J. B.; DE CARVALHO, N. R.; STECKLING, F. M.; DE VARGAS, L. S.; COURTES, A. A.; STEFANELLO, S. T.; MARTINS, C. C.; BRESCIANI, G.; DOS SANTOS, D. L.; SOARES, F. A. A. An active lifestyle induces positive antioxidant enzyme modulation in peripheral blood mononuclear cells of overweight/obese postmenopausal women. Life Sciences, 2014. Disponível em: <http://www.sciencedirect.com/science/article/pii/ S0024320514009436 >. Acesso em: 01 ago. 2015.

HETLAND, G.; JOHNSON, E.; LYBERGÀ, T.; BERNARDSHAW, S.; TRYGGESTAD, A. M. A.; GRINDE, B. Effects of the Medicinal Mushroom Agaricus blazei Murill on Immunity, Infection and Cancer. Scandinavian Journal of Immunology, 2008. Disponível em: <http://onlinelibrary.wiley.com/doi/10.1111/j. 1365-3083.2008.02156.x/epdf > . Acesso em: 18ago. 2015.

IBGE - Instituto Brasileiro de Geografia e Estatística. Pirâmide Etária Absoluta Brasileira. IBGE, 2011. Disponível em: <http://www.ibge.gov.br/home/estatistica/populacao/projecao_da_populacao/2008/piramide/piramide.shtm>. Acesso em: 22 ago. 2015.
LEBRÃO, M. L.; DUARTE, Y. A. de 0 (Org.). SABE: saúde, bem-estar e envelhecimento. 0 Projeto Sabe no município de São Paulo. Brasília, DF: Organização Pan-Americana da Saúde, 2006.

LIMENIS, E.; GROSBEIN, H. A.; FELDMAN, B. M. The relationship between physical activity levels and pain in children with juvenile idiopathic arthritis. The Journal of Rheumatology, fevereiro de 2014. Disponível em: <http://www.jrheum.org/content/41/2/345.full.pdf+html>. Acesso em: 22 ago. 2015.

MARINHO, R.; MEKARY, R. A.; MUÑOZ, V. R.; GOMES, R. J.; PAULI, J. R.; DE MOURA, L. P. Regulation of hepatic TRB3/Akt interaction induced by physical exercise and its effect on the hepatic glucose production in an insulin resistance state. Diabetol Metab Syndr, 2015. Disponível em: <http://www.dmsjournal.com/content/ pdf/s13098-015-0064-x.pdf >. Acesso em: 22 ago. 2015.

MIRANDA, A.M.; RIBEIRO, G.M.; CUNHA, A.C. et al. Hypolipidemic effect of the edible mushroom Agaricus blazei in rats subjected to a hypercholesterolemic diet. J Physiol Biochem, 2014. Disponível em: <http:// link.springer.com/article/10.1007\%2Fs13105013-0295-y>. Acesso em: 18 ago. 2015.

PAOLI, A.; BIANCO, A. What Is Fitness Training? Definitions and Implications: A Systematic Review Article. Iran J Public Health, maio de 2015. Disponível em: <http://ijph.tums.ac.ir/>. Acesso em: 22 dez. 2015.

PENTECOST, C.; FARRAND, P.; GREAVES, C.J.; TAYLOR, R. S.; WARREN, F. C.; HILLSDON, M.; GREEN, C.; WELSMAN, J. R.; RAYSON, K.; EVANS, P. H.; TAYLOR, A. H. Combining behavioural activation with physical activity promotion for adults with depression: findings of a parallel-group pilot randomised controlled trial (BacPAc). Trials, 2015. Disponível em: <http://www.trialsjournal. com/content/16/1/367>. Acesso em: 22 dez. 2015.

RÉGIS, W. C. B.; VAL, C. H.; BRANT, F.; MIRANDA, A. S.; RODRIGUES, F. G.; OLIVEIRA, B. C. L.; SANTOS, E. A.; ASSIS, D. R. R.; ESPER, L.; SILVA, B. C.; RACHID, 
M. A.; TANOWITZ, H. B.; TEIXEIRA, A. L.; TEIXEIRA, M. M.; MACHADO, F. S. Effect of mushroom Agaricus blazei on immune response and development of experimental cerebral malaria. Malaria Journal, 2015 Disponível em: <http://www.malariajournal.com/content/14/1/311>. Acesso em: 18 ago. 2015.

ROWISKI, R.; KOZAKIEWICZ, M.; KDZIORA-KORNATOWSKA, K.; HÜBNER-WONIAKA, E.; KDZIORAB, J. Markers of oxidative stress and erythrocyte antioxidant enzyme activity in older men and women with differing physical activity. Experimental Gerontology, 2013. Disponivel em: <http://www.sciencedirect.com/ science/article/pii/S0531556513002313>. Acesso em: 19 ago. 2015.

SALEHI, A.; HARRIS, N.; SEBAR, B.; COYNE, E. Self-Perception of Quality of Life and Its Association with Lifestyle Behaviours of Young Iranian Women. Iran J Public Health, março de 2015. Disponível em: <http:// www.ncbi.nlm.nih.gov/pmc/articles/PMC4402411/ pdf//JPH-44-332.pdf>. Acesso em: 22 ago. 2015.

SHIROMA, E. J.; LEE, I-M. Physical Activity and Cardiovascular Health Lessons Learned From Epidemiological Studies Across Age, Gender, and Race/Ethnicity. Circulation, 2010. Disponível em: <http://circ. ahajournals.org/content/122/7/743. full.pdf+html>. Acesso em: 22 ago. 2015.

TANIGAWA, T.; TAKECHI, H.; ARAl, H.; YAMADA, M.; NISHIGUCHI, S.; AOYAMA, T. Effect of physical activity on memory function in older adults with mild Alzheimer's disease and mild cognitive impairment. Geriatr Gerontol Int, março de 2014. Disponível em: <http:// onlinelibrary.wiley.com/doi/10.1111/ggi.12159/abstract.> Acesso em 22 ago. 2015.

WANG, H.; LI, G.; ZHANG, W.; HAN, C.; XU, X.; Li, YONG, Y.-P. The Protective Effect of Agaricus blazei Murrill, Submerged Culture Using the Optimized Medium Composition, on Alcohol-Induced Liver Injury. BioMed Research International, 2014. Disponivel em: $<$ http:// www.hindawi.com/journals/bmri/2014/573978/>. Acesso em 18 ago. 2015.
Recebido em: 16 de março de 2017 Aceito em: 11 de maio de 2017 